\title{
AVALIAÇÃO DA TAXA DE REMOÇÃO E CUSTO DO TRATAMENTO DA ÁGUA RESIDUÁRIA DO CAFÉ POR UM SISTEMA ELETROLÍTICO
}

\author{
Benvindo Sirtoli Gardiman Junior" \\ Giovanni de Oliveira Garcia* \\ Edvaldo Fialho dos Reis ${ }^{* * *}$
}

\begin{abstract}
RESUMO: A Água Residuária do Café (ARC) pode causar sérios impactos ambientais se for lançada no meio sem tratamento prévio. Assim, o tratamento eletrolítico surge como uma técnica bastante promissora nesse procedimento. Por esse motivo, objetivou-se nesse estudo avaliar a taxa de remoção dos poluentes e o custo do tratamento por essa técnica com base nas variáveis turbidez, sólidos totais e Demanda Química de Oxigênio (DQO) e no comportamento do $\mathrm{pH}$ e da condutividade elétrica. Utilizou-se uma célula eletrolítica com as dimensões internas de 8,15 de largura, 13,8 $\mathrm{cm}$ de comprimento e $8,9 \mathrm{~cm}$ de profundidade, possuindo um volume de $1000 \mathrm{~cm}^{3}$. Placas de alumínio com área de $0,02054 \mathrm{~m}^{2}$ foram empregadas em uma configuração monopolar em paralelo. Realizaram-se ensaios em batelada variando a distância de placas, densidade de corrente e intervalos de coleta do efluente do reator entre 60 a 1440 segundos para a água residuária com e sem reúso, respectivamente. Por fim, constatou-se que os poluentes apresentam taxas e custos de remoção específicos, estando relacionados à corrente aplicada, distância de placas, eficiência de remoção, tempo de detenção hidráulico e concentração de outros componentes.
\end{abstract}

PALAVRAS-CHAVE: Água Residuária do Café; Contaminação ambiental; Eletroflotação.

\section{REMOVAL RATE AND TREATMENT COSTS OF COFFEE RESIDUE WATER BY AN ELECTROLITIC SYSTEM}

ABSTRACT: Coffee Residue Water (CRW) may cause serious environmental impacts if deposited in the environment without any treatment. Electrolithic treatment is a

\footnotetext{
"Docente MSc Instituto Federal do Espírito Santo, Campus Ibatiba, Coordenadoria de Meio Ambiente, Brasil. E-mail: benvindo.gardiman@ifes.edu.br

** Docente DSc Universidade Federal do Espírito Santo. Departamento de Engenharia Rural, Campus de Alegre, Brasil.

**** Docente DSc Universidade Federal do Espírito Santo. Departamento de Engenharia Rural, Campus de Alegre, Brasil.
} 
highly promising technique for this procedure. Current analysis evaluates removal rate of pollutants and treatment costs of the technique, based on variables turbidity, total solids and Chemical Oxygen Demand (COD), coupled to $\mathrm{pH}$ and electric conductivity behavior. An electrolithic cell, measuring 8.15 width, $13.8 \mathrm{~cm}$ length and $8.9 \mathrm{~cm}$ depth, with a volume of $1000 \mathrm{~cm}^{3}$, was employed. Aluminum plates, $0.02054 \mathrm{~m}^{2}$, were used for a parallel monopolar configuration. Batch assays were employed with variations in plate distance, current density and intervals in the effluent collection of the reactor between 60 and $1440 \mathrm{sec}$. for residue water, with and without reuse, respectively. Pollutants had specific removal rates and costs, related to applied current, plate distance, removal efficiency, hydraulic detention time and the concentration of other components.

KEY WORDS: Coffee residue water; Environmental contamination; Electroflotation.

\section{INTRODUÇÃO}

O café é um dos produtos mais importantes, representando grande expressividade na economia brasileira (CAMPOS et al., 2014). O valor estipulado pelo mercado internacional é determinado pela qualidade do grão, que por sua vez está em função das características inerentes aos frutos (CAMPOS et al., 2010), podendo estar relacionado diretamente com a forma que foram processados (BRANDÃO et al., 2016).

Visando agregar valor ao produto, após a colheita os grãos são descascados e desmucilados via úmida, gerando a Água Residuária do Café (ARC) (SILVA et al., 2014). Esse efluente apresenta alto teor de matéria orgânica, expressa em Demanda Bioquímica de Oxigênio (DBO 5,20 으) e Demanda Química de Oxigênio (DQO), é rica também em açúcares, proteínas, amido, pectina, compostos fenólicos, nitrogênio total, fósforo total, sódio (CAMPOS et al., 2010) e, principalmente, potássio que, se lançada sem tratamento em corpos hídricos, pode causar diversos impactos ambientais (FIA et al., 2010d; 2010e; OLIVEIRA; BRUNO, 2013).

Neste sentido, pesquisadores propõem diversos sistemas de tratamento da ARC, desde a decantação (SILVA et al., 2014), sistemas biológicos (FIA et al., 2010a; OLIVEIRA; BRUNO, 2013), químicos (KONDO et al., 2014), etc. Contudo, alguns 
métodos podem apresentar elevados tempos de detenção, consumo de produtos químicos, difícil implantação e manutenção e grande área necessária. Assim, o tratamento eletrolítico surge como uma alternativa bastante promissora (AQUINO NETO; ANDRADE, 2009).

A técnica de eletrofloculação apresenta vantagens quando comparada aos métodos convencionais de tratamento de efluentes, tais como: alta eficiência na remoção de partilhados, sistema compacto, custos relativamente baixos, possibilidade de automação (CERQUEIRA et al., 2014), menor tempo de detenção hidráulica (TDH), redução ou ausência de produtos químicos e microrganismos adicionados, rápida sedimentação dos flocos produzidos (CHEN, 2004; KOBYA et al., 2006a; CERQUEIRA et al., 2009; KABDAŞLI et al., 2012). Entretanto, apresenta consumo de energia, desgaste das placas do metal usado e passivação dos eletrodos quando aplicados longos TDH's (KOBYA et al., 2006b).

Assim, objetivou-se nesse estudo avaliar a taxa de remoção dos poluentes refletidos em termos de turbidez, sólidos totais e demanda química de oxigênio e o custo geral do tratamento da Água Residuária do Café (ARC) com e sem recirculação.

\section{MATERIAL E MÉTODOS}

AÁgua Residuária do Café (ARC) oriunda do despolpamento e desmucilagem dos grãos de café da espécie Arábica de uma unidade de processamento sem recirculação (ARC1) e com cerca de 8 recirculações (ARC2). O experimento foi implantado e conduzido no Laboratório de Pesquisa do Instituto Federal de Ciência e Tecnologia do Espírito Santo (Ifes), Campus Ibatiba.

As ARC's foram acondicionadas em garrafas de 2 L e armazenadas sob temperatura a $4{ }^{\circ} \mathrm{C}$, garantindo a preservação das características (ABNT, 1987; MATOS et al., 2007; 2015). As variáveis DQO, fósforo, nitrogênio e sólidos totais foram determinadas seguindo o estabelecido pelo Standard Methods (APHA, 2012). Já o teor de açúcares totais foi determinado conforme Albarici et al. (2009); potássio pela NBR 13810 (ABNT, 1997); fenóis totais pelo método de Folin Ciocalteau; e os demais por aparelhos específicos devidamente calibrados: condutivímetro, fotômetro Hanna (colorímetro) e pHmetro. 
Para otimizar o monitoramento do sistema, utilizaram-se as variáveis mais representativas do principais poluentes existente no efluente, tais como turbidez, representados os sólidos suspensos; os sólidos totais, para todos os sólidos presentes; a DQO, o consumo de oxigênio para degradação da matéria total e açúcares, o pH para variação dos íons $\mathrm{H}+\mathrm{e} \mathrm{OH}$ - gerados e condutividade elétrica para os sais presentes (CETESB, 2008).

A célula de eletrofloculação foi construída em vidro conforme os critérios estabelecidos por Mollah et al. (2004), com as dimensões internas: largura de 8,15 $\mathrm{cm}$, comprimento de $13,8 \mathrm{~cm}$ e profundidade de $8,9 \mathrm{~cm}$, possuindo um volume de $1000 \mathrm{~cm}^{3}$ (Figura 1).

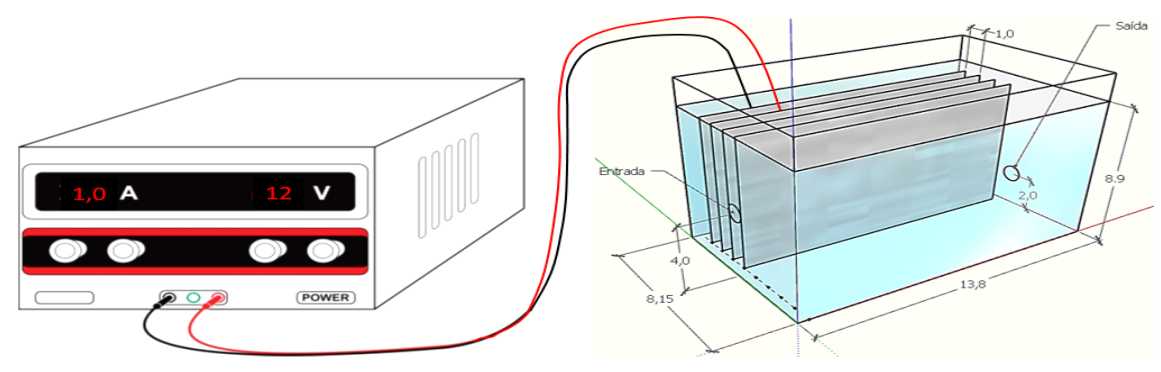

Figura 1. Representação esquemática do Reator eletrólito com a fonte elétrica (Fonte: O Autor).

Os eletrodos foram confeccionados em chapa de alumínio (ABNT/ ASTM 1050) medindo 0,8 $\mathrm{mm}$ de espessura, 7,9 cm de largura por $13,0 \mathrm{~cm}$ de comprimento, com área superficial de $0,02054 \mathrm{~m}^{2}$, ficando totalmente mergulhados no efluente. A configuração utilizada foi a monopolar em paralelo, onde os eletrodos são polarizados intercaladamente através de ligações externas, sendo os anodos os eletrodos de sacrifício (MOLLAH et al., 2004).

Os ensaios em batelada foram realizados combinando os seguintes fatores: Distância de Placas (DP) nos níveis de 10, 20 e 30 mm; Densidade de Corrente (DC) 
em $100,75,50$ e $25 \mathrm{~A} \mathrm{~m}^{-2}$ e os intervalos entre as coletas das amostras no reator variando entre 60 a 1440 segundos (Tabela 1 ). A variação entre os intervalos de coletas fez-se necessário tendo em vista que o aumento da corrente e a diminuição da distância entre placas proporcionam um incremento de metal oxidado, melhorando a eficiência do processo e consequentemente diminuindo o tempo operacional (CARMONA et al., 2006).

Tabela 1. Distâncias de Placas (DP), Densidades de Corrente (DC) aplicadas e intervalos de coletas para cada um dos experimentos realizados para tratamento da Água Residuária do Café sem (ARC1) e com (ARC2) recirculação

\begin{tabular}{ccccc}
\hline Ensaios & DP & DC & \multicolumn{2}{c}{ Intervalos de coleta (s) } \\
& $\mathrm{mm}$ & $\mathrm{A} \mathrm{m}^{-2}$ & ARC1 & ARC2 \\
\hline 1 & 10 & 100 & 60 & 180 \\
2 & 10 & 75 & 80 & 360 \\
3 & 10 & 50 & 100 & 900 \\
4 & 10 & 25 & 120 & 1200 \\
5 & 20 & 100 & 60 & 480 \\
6 & 20 & 75 & 60 & 600 \\
7 & 20 & 50 & 60 & 960 \\
8 & 20 & 25 & 120 & 1200 \\
9 & 30 & 100 & 120 & 480 \\
10 & 30 & 75 & 120 & 300 \\
11 & 30 & 50 & 120 & 720 \\
12 & 30 & 25 & 240 & 1440 \\
\hline
\end{tabular}

A taxa de remoção média (TRM) foi calculada com base na remoção ou alteração média das variáveis nos intervalos de coleta, conforme a equação (1):

$\operatorname{TRM}=\overline{\mathrm{X}_{\mathrm{t}_{0}}} \frac{\left(\mathrm{C}_{\mathrm{t}_{0}}-\mathrm{C}_{\mathrm{t}_{1}}\right)}{\left(\mathrm{t}_{1}-\mathrm{t}_{0}\right)}$

Em que:

TRM, Taxa de Remoção Média em NTU $\min ^{-1}$ ou mg L ${ }^{-1} \min ^{-1}$ ou dS $\mathrm{m}^{-1} \mathrm{~min}^{-1}$ ou unidades de $\mathrm{pH}$ minn $^{-1}$;

$\overline{\mathrm{X}} \overline{\mathrm{X}}$ é a média das taxas de remoção ou alteração das variáveis analisadas nos intervalos de tempo $\left(\mathrm{t}_{0} \mathrm{a} \mathrm{t}_{\mathrm{f}}\right)$, em minutos; 
C é a concentração da variável estudada no tempo, em NTU, $\mathrm{mg} \mathrm{L}^{-1}$ ou dS $\mathrm{m}^{-1}$;

t é o tempo em que a variável foi analisada, sendo $t_{0}$ antes do experimento, $\mathrm{t}_{1}$ a primeira coleta após intervalo de t minutos, e assim sucessivamente até $\mathrm{t}_{\mathrm{f}}$ o final.

O consumo de energia elétrica específico (CEE) foi calculado, conforme Asha e Kumar (2015a), considerando as concentrações iniciais e finais dos compostos removidos da $\mathrm{ARC}$ nos diferentes tempos de tratamento avaliados (Equação 2):

$$
\mathrm{CEE}=\frac{V \times I \times t \times 1000}{60 \times\left(C_{0}-C_{t}\right) \times S V}
$$

Para a qual:

CEE, Consumo de Energia Elétrica Específico em kWh $1000^{-1}$ unidades dos compostos removidos ( $\mathrm{kWh} \mathrm{kg}^{-1}$ para ST's e DQO; e $\mathrm{kWh} \mathrm{kNTU}^{-1}$ para turbidez);

V é a tensão aplicada, em Volts;

I é a corrente aplicada, em Ampère;

t é o tempo de eletrólise, em minutos;

Sv é o volume de efluente tratado em litros; e

$\mathrm{C}_{\mathrm{o}}$ e $\mathrm{C}_{\mathrm{t}}$, a concentração inicial e final, respectivamente no tempo avaliado.

O consumo de energia elétrica geral (CEG), que representa o custo de operação no tempo estabelecido em um reator de batelada (HOLT et al., 2005; KOBYA et al., 2006; MOLLAH et al., 2004), foi obtido conforme a Equação 3:

$$
\mathrm{CEG}=\frac{\mathrm{V} \times \mathrm{I} \times \mathrm{t}}{60 \times \mathrm{SV}}
$$

Em que:

CEG, Consumo de Energia Elétrica Geral em $\mathrm{kWh} \mathrm{m}^{-3} \mathrm{ou} \mathrm{Wh} \mathrm{dm}^{-3}$;

V é a tensão aplicada, em Volts;

I é a corrente aplicada, em Ampère;

t é o tempo de eletrólise, em minutos; e

Sv é o volume de efluente tratado em litros.

A massa de eletrodo consumido (Mel) é definido matematicamente pela 
equação 4 (HOLT et al., 2005; KUOKKANEN et al., 2013; MOLLAH et al., 2004):

$$
\text { Mel }=\frac{\mathrm{I} \times \mathrm{t} \times \mathrm{M}}{\mathrm{Z} \times \mathrm{F}}
$$

Onde:

mel = quantidade máxima do eletrodo consumida, em g;

$\mathrm{I}=$ corrente aplicada, em A;

$\mathrm{t}=$ tempo de aplicação da corrente, em s;

$\mathrm{M}=$ massa molar do material do eletrodo, $\mathrm{M}_{\mathrm{Al}}=26,982 \mathrm{~g} \mathrm{~mol}^{-1}$;

$\mathrm{z}=$ número de elétrons envolvidos na reação de oxidação do elemento do ânodo, $\mathrm{z}_{\mathrm{Al}}=3$;

$\mathrm{F}=$ constante de Faraday, $96.485 \mathrm{C} \mathrm{mol}^{-1}$.

O custo de operação do sistema pode ser calculado de acordo com a equação 5 (KOBYA et al., 2006; KUOKKANEN et al., 2013):

$$
\mathrm{C}_{\text {Operação }}=(\mathrm{a} \times \mathrm{Mel})+(\mathrm{b} \times \mathrm{CEG})
$$

$\mathrm{C}_{\text {operação }}=$ Custo de operação, $\mathrm{R} \$ \mathrm{~m}^{-3}$ efluente tratado;

$\mathrm{a}=$ custo mássico do metal usado na placa, $\mathrm{R} \$ \mathrm{~kg}^{-1}$ do alumínio (CEMPRE, 2017);

Mel = Massa consumida do eletrodo, $\mathrm{kg} \mathrm{m}^{-3}$ efluente tratado;

$\mathrm{b}=$ Custo de energia, $\mathrm{R} \$ \mathrm{kWh}^{-1}$ (ESCELSA, 2017);

$\mathrm{CEG}=$ Consumo de Energia Geral, $\mathrm{kWh} \mathrm{m}^{-3}$ efluente tratado.

\section{RESULTADO E DISCUSSÃO}

Os resultados da caracterização das águas residuárias do café (ARC1 e ARC2) utilizadas nos tratamentos seguem apresentados na Tabela 2. Observa-se que os valores para a ARC1 estão próximos aos encontrados por Eustáquio et al. (2014), Matos et al. (2015), Selvamurugam et al. (2010a) e Ulavi e Kumar (2013) e ARC2 remetem a Campos et al. (2010) e Peralta et al. (2014), quando estudaram o referido efluente. 
Tabela 2. Caracterização físico-química da água residuária do café sem recirculação (ARC1) e com recirculação (ARC2) utilizadas no estudo

\begin{tabular}{|c|c|c|c|}
\hline \multirow{2}{*}{ Variável } & \multirow{2}{*}{ Unidade } & \multicolumn{2}{|c|}{ Valores Médios } \\
\hline & & ARC1 & ARC2 \\
\hline Açúcares & $\mathrm{mg} \mathrm{L}^{-1}$ & $6.950 \pm 36,5$ & $17.984 \pm 469$ \\
\hline $\mathrm{CE}$ & $\mathrm{dS} \mathrm{m}^{-1}$ & $1,43 \pm 0,45$ & $2,84 \pm 0,76$ \\
\hline Cor & PCU & $3.610 \pm 193$ & $6.670 \pm 146$ \\
\hline DQO & $\mathrm{mg} \mathrm{L}^{-1}$ & $26.800 \pm 8.725$ & $47.420 \pm 9.228$ \\
\hline $\mathrm{CF}$ & $\mathrm{mg} \mathrm{L}^{-1}$ & $212 \pm 8,1$ & $542 \pm 58$ \\
\hline K & $\mathrm{mg} \mathrm{L}^{-1}$ & $471,44 \pm 55$ & $1.354 \pm 105$ \\
\hline $\mathrm{Nt}$ & $\mathrm{mg} \mathrm{L}^{-1}$ & $12,2 \pm 0,8$ & $40,1 \pm 1,2$ \\
\hline OD & $\mathrm{mg} \mathrm{L}^{-1}$ & $5,1 \pm 0,3$ & $1,4 \pm 0,4$ \\
\hline $\mathrm{pH}$ & -.-- & $4,7 \pm 0,4$ & $4,43 \pm 0,2$ \\
\hline Pt & $\mathrm{mg} \mathrm{L}^{-1}$ & $16 \pm 1,2$ & $20,1 \pm 2,0$ \\
\hline ST & $\mathrm{mg} \mathrm{L}^{-1}$ & $15.818 \pm 7.184$ & $21.998 \pm 8.270$ \\
\hline Turbidez & NTU & $1.008 \pm 141$ & $2.369 \pm 360$ \\
\hline
\end{tabular}

No tratamento de efluentes a avaliação da taxa de remoção é um parâmetro muito importante na tomada de decisões. Assim, por meio da Tabela 3 detectase que as variáveis turbidez e sólidos totais (ST) apresentam-se maiores taxas de remoção média, em NTU min $^{-1}$ e $\mathrm{mg} \mathrm{L}^{-1}$, respectivamente, quando aplicados maiores níveis de DC em todas as DP's estudadas. Elevados níveis de DC conduzem à maior geração de gases na forma de hidrogênio $\left(\mathrm{H}_{2}\right)$ (KUOKKANEN et al., 2013), proporcionando a flotação dos sólidos, principalmente os suspensos que causam a turbidez, corroborando com a relação evidenciada por Valente et al. (2015) para efluentes de laticínio.

Para água residuária com recirculação (ARC2) observa-se uma tendência similar (Tabela 4), variando as maiores taxas de remoção apenas entre 100 e $75 \mathrm{~A} \mathrm{~m}^{-2}$. As alegações dos autores citados anteriormente são válidas para esse caso também. 
Tabela 3. Taxa de remoção média de turbidez, Sólidos Totais (ST), Demanda Química de Oxigênio (DQO), Condutividade Elétrica (CE) e pH no Tempo de Detenção Hidráulico (TDH) para a Água Residuária do Café (ARC1) sem recirculação

\begin{tabular}{ccccccccc}
\hline \multirow{2}{*}{ Ensaios } & $\begin{array}{c}\text { DP } \\
\mathrm{mm}\end{array}$ & $\begin{array}{c}\text { DC } \\
\mathrm{A} \mathrm{m}^{-2}\end{array}$ & $\begin{array}{c}\text { TDH } \\
\mathrm{min}\end{array}$ & $\begin{array}{c}\text { Turbidez } \\
\mathrm{NTU} \mathrm{min}^{-1}\end{array}$ & $\begin{array}{c}\text { ST } \\
\mathrm{mg} \mathrm{L}^{-1}\end{array}$ & $\begin{array}{c}\text { DQO } \\
\mathrm{min}^{-1}\end{array}$ & $\begin{array}{c}\text { CE } \\
\mathrm{dS} \mathrm{cm}^{-1}\end{array}$ & $\begin{array}{c}\text { pH } \\
\mathrm{min}^{-1}\end{array}$ \\
\hline 1 & 10 & 100 & 4,12 & 150,67 & 1359,51 & 636,59 & $-0,03$ & 0,17 \\
2 & 10 & 75 & 6,87 & 106,56 & 403,20 & 298,15 & 0,00 & 0,11 \\
3 & 10 & 50 & 7,00 & 116,79 & 226,36 & 595,00 & 0,01 & 0,11 \\
4 & 10 & 25 & 7,44 & 81,84 & 243,74 & 595,00 & 0,01 & 0,09 \\
5 & 20 & 100 & 4,12 & 201,85 & 593,35 & 528,16 & $-0,04$ & 0,15 \\
6 & 20 & 75 & 4,12 & 174,66 & 325,74 & 527,65 & $-0,04$ & 0,05 \\
7 & 20 & 50 & 4,12 & 140,40 & 467,43 & 709,30 & 0,01 & 0,06 \\
8 & 20 & 25 & 12,62 & 69,55 & 53,17 & 377,97 & 0,00 & 0,03 \\
9 & 30 & 100 & 8,24 & 108,02 & 93,86 & 694,18 & $-0,01$ & 0,07 \\
10 & 30 & 75 & 8,24 & 106,16 & 69,86 & 514,99 & 0,00 & 0,06 \\
11 & 30 & 50 & 10,41 & 83,74 & 23,56 & 745,93 & 0,01 & 0,03 \\
12 & 30 & 25 & 29,78 & 32,16 & 28,09 & 110,61 & 0,00 & 0,01 \\
\hline
\end{tabular}

Tabela 4. Taxa de remoção média de turbidez, Sólidos Totais (ST), Demanda Química de Oxigênio (DQO), Condutividade Elétrica (CE) e pH no Tempo de Detenção Hidráulico (TDH) para a Água Residuária do Café (ARC2) com recirculação

\begin{tabular}{ccccccccc}
\hline \multirow{2}{*}{ Ensaios } & $\begin{array}{c}\text { DP } \\
\mathrm{mm}\end{array}$ & $\begin{array}{c}\text { DC } \\
\mathrm{A} \mathrm{m}^{-2}\end{array}$ & $\begin{array}{c}\text { TDH } \\
\mathrm{min}\end{array}$ & $\begin{array}{c}\text { Turbidez } \\
\mathrm{NTU} \mathrm{min}^{-1}\end{array}$ & $\begin{array}{c}\text { ST } \\
\mathrm{mg} \mathrm{L}^{-1} \mathrm{~min}^{-1}\end{array}$ & $\begin{array}{c}\text { DQO } \\
\mathrm{dS} \mathrm{m} \mathrm{m}^{-1}\end{array}$ & $\begin{array}{c}\text { pH } \\
\text { uni pH } \mathrm{min}^{-1}\end{array}$ \\
\hline 1 & 10 & 100 & 22,34 & 105,79 & 250,88 & 465,37 & $-0,02$ & 0,04 \\
2 & 10 & 75 & 54,10 & 35,18 & 116,23 & 144,12 & 0,00 & 0,02 \\
3 & 10 & 50 & 101,12 & 18,12 & 74,12 & 169,30 & 0,01 & 0,01 \\
4 & 10 & 25 & 160,92 & 11,20 & 43,10 & 112,91 & 0,00 & 0,01 \\
5 & 20 & 100 & 50,53 & 46,38 & 91,62 & 185,96 & 0,01 & 0,02 \\
6 & 20 & 75 & 54,95 & 38,99 & 132,07 & 114,70 & 0,00 & 0,02 \\
7 & 20 & 50 & 50,53 & 39,47 & 50,90 & 218,83 & 0,01 & 0,01 \\
8 & 20 & 25 & 109,89 & 19,87 & 37,07 & 72,76 & 0,00 & 0,01 \\
9 & 30 & 100 & 34,41 & 64,94 & 130,19 & 292,47 & 0,03 & 0,04 \\
10 & 30 & 75 & 20,62 & 110,04 & 97,03 & 159,02 & 0,02 & 0,03 \\
11 & 30 & 50 & 37,89 & 59,71 & 77,57 & 62,44 & 0,01 & 0,02 \\
12 & 30 & 25 & 161,80 & 11,86 & 19,41 & 58,69 & 0,00 & 0,02 \\
\hline
\end{tabular}


Em tempo, verifica-se que há uma limitação na remoção de turbidez nos TDH's indicados, como pode ser apreciado nas Tabelas 3 e 4. Essa limitação se deve, principalmente, pela excessiva geração de hidróxidos que deixa a solução com uma coloração esbranquiçada, interferindo na passagem que luz e consequentemente conferindo uma maior turbidez (BARBOSA; TERAN, 2009). O incremento de hidróxidos ocorre, mais acentuadamente, quando aplicados maiores níveis de DC's e menores de DP's, dissolvendo o metal do anodo de acordo com a Lei de Faraday (KUOKKANEN et al., 2013).

O aparecimento inesperado desse aspecto também foi observado por Valente (2012), principalmente quando aplicados maiores níveis de tempo e densidade de corrente para tratar efluente de laticínios com eletrodos de zinco. Isso sugere que tais DC's aplicadas podem estar acima da necessidade para o tratamento, podendose deduzir que atingiu-se o limite de eficiência de remoção dos poluentes por esse sistema, nessas condições, acima desse tempo. Pode também citar que acima desses TDH's há um maior consumo de energia e consumo de eletrodos (KUOKKANEN et al., 2013).

Assim, pressupõe-se que tempos superiores aos apresentados neste estudo podem inviabilizar o tratamento por meio eletrolítico, o que contradiz algumas pesquisas que avaliam apenas a eficiência final do tratamento e não o desempenho do tratamento em intervalos de tempo. A paralisação do tratamento ao detectar a presença de flocos brancos também foi realizada por alguns autores (BARBOSA; TERAM, 2009; COMBATT et al., 2017), indicando que a remoção de sólidos havia cessado.

Para o $\mathrm{pH}$, analogamente ao verificado para a turbidez, houve maiores incrementos, em termos de unidades de $\mathrm{pH}$ min $^{-1} \mathrm{em}$ DC's mais altas nas duas ARC's tratadas. Essa elevação se deve, por certo, a maior geração de hidroxilas, produtos da hidrólise, que acontece no cátodo (KUOKKANEN et al., 2013). A elevação do pH já foi observada por outros autores (BENAZZI et al., 2015; KANNAN et al., 2006; KOBYA et al., 2006b; VALENTE et al., 2015) e acontece linearmente até o fim do tratamento.

Corroborando com essa tendência, os ensaios conduzidos com menores DC's (25 A m²), menores TDH (min.) e maior DP (30 mm), exibiram menores incrementos de $\mathrm{pH}$ e taxas de remoção mais sofríveis para a turbidez e ST em ambas 
as ARC's, justificando que a geração de produtos no tratamento eletrolítico, como hidróxidos e os gases, seguem a lei de Faraday (HOLD et al., 2005).

A taxa de remoção de DQO condiz com o apresentado pela turbidez e ST, se comparado apenas as DC's maiores e menores dentro das mesmas DP's. Entretanto, nota-se, inesperadamente, que os ensaios 7 e 11 da ARC1 alcançaram valores de 709,30 e $745,93 \mathrm{mg} \mathrm{L}^{-1} \mathrm{~min}^{-1}$ de taxa de remoção, respectivamente. Do mesmo modo o ensaio $7 \mathrm{da} \mathrm{ARC} 2$ obteve, à $50 \mathrm{~A} \mathrm{~m}^{-2}$, taxa de remoção acima do apresentado por $100 \mathrm{~A} \mathrm{~m}^{-2}$ na mesma DC. O observado reforça os indícios de que o mecanismo de remoção de DQO por tratamento eletrolítico ainda não é totalmente compreendido, principalmente quando se têm presentes espécies iônicas e compostos solúveis no efluente (CASSILAS et al., 2007). Relata-se também que efluentes com elevada concentração de compostos orgânicos dissolvidos, açúcares e carboidratos de alta solubilidade em água, como a ARC (CAMPOS et al., 2010), apresentam baixas eficiências de remoção de DQO por eletrofloculação (BENAZZI et al., 2016; CASSILAS et al., 2007).

As menores remoções de DQO foram evidenciadas em menores DC's, maiores DP's e menores tempos, como o ensaio 12. Essa tendência foi relatada por Tchamango et al. (2010) no tratamento eletroquímico, em modo batelada, de um efluente sintético de leite e Chen et al. (2000), no tratamento eletroquímico, em modo batelada, de efluentes de restaurante. Ambos os autores atribuíram esse comportamento, em relação a DQO, à presença de compostos orgânicos dissolvidos, os quais são de difícil remoção por processos que empregam a coagulação como forma de separação (BENAZZI et al., 2016; VALENTE et al., 2015; VALENTE et al., 2012).

A complexidade do comportamento da variável DQO, neste estudo, contradiz o observado por alguns autores. Elevados TDH's contribuem para maior remoção da DQO, em efluentes de curtume (SONG et al., 2004), óleo de oliva (INAN et al., 2004), de corante azul (GOLDER et al., 2005). Contudo, observou-se não haver tal relação, ao contrário, uma menor remoção foi evidenciada nos ensaios que apresentaram maiores THD's. $\mathrm{O}$ apreciado confirma a hipótese de que a remoção da DQO ocorre por processos de oxidação eletroquímica (KABDAŞLI et al., 2012) que demandam elevados TDH's, DP's e DC's para que todas as reações eletroquímicas aconteçam. 
Asha e Kumar (2015a), usando eletrodos de alumínio para tratar ARC na Índia, reportam que obtiveram uma baixa eficiência de remoção (30\%) de DQO nos primeiros 15 minutos de tratamento. Os autores atribuem o fato à evolução do hidrogênio no cátodo ainda não ter iniciado. Destacaram, porém, que após 60 minutos de eletrólise a taxa de remoção de DQO foi próxima de $90 \%$ e de até 93\%, em 75 minutos. Entretanto, é válido ressaltar que os autores não avaliaram o comportamento da turbidez e nem a formação de hidróxidos que poderiam inviabilizar o tratamento.

A remoção de DQO por esse sistema ainda não está completamente elucidado (VALENTE, 2012). Todavia, sabe-se que a interação entre as partículas colidais e dispersas presentes no efluente podem influenciar na avaliação, havendo poucos dados publicados, o que reforça a necessidade de pesquisas envolvendo esses fatores intervenientes.

A condutividade elétrica (CE) apresentou incrementos negativos na média, demonstrando uma perda de condutividade do efluente, principalmente para os ensaios com maior DC aplicada e menores TDH's. Cetesb (2008) cita que a condutividade elétrica está ligada diretamente à quantidade de sólidos dissolvidos totais da solução e dando uma noção da quantidade de sais presentes no efluente. Isso permite induzir que a remoção dos sólidos, como também evidenciado na turbidez, pode ter contribuído para diminuir a CE. Por outro lado, destaca-se que longos TDH's e altas DC's podem contribuir para altear os teores de CE pela incorporação de íons metálicos na solução (CHEN et al., 2000), relação inversa observada neste estudo quando se relaciona $\mathrm{pH}$ e $\mathrm{CE}$.

$\mathrm{O}$ consumo de energia elétrica geral e específico para remoção dos compostos presentes na ARC1 e ARC2 encontra-se apresentado na Tabela 5. Nela observa-se que maiores consumos gerais, dentro das mesmas DP's, estão associados a maiores níveis de correntes (Corr) e TDH's aplicados. Ao se analisar o custo de remoção da turbidez, tem-se que o ensaio 4 da ARC1 (Condição: DP: 10 mm; Corr: 0,5 A; TDH: 7,44 min.) apresentou o menor consumo elétrico $\left(\mathrm{kWh} \mathrm{kNTU}^{-1}\right)$. Para a ARC2 o ensaio 10 (Condição: DP: $30 \mathrm{~mm}$; Corr: 1,5 A; TDH: 20,62 min.) esboçou menor custo geral: $6,19 \mathrm{kWh} \mathrm{m}^{-3}$. Já para ST e DQO, evidenciou-se um melhor custo benefício no ensaio 1 (DP: $10 \mathrm{~mm}$; Corr: 2,0 A; TDH: 4,12 min.) e ensaio 7 
(Condição: DP: 20 mm; Corr: 1,0 A; TDH: 4,12 min.), respectivamente, para a ARC1 e similarmente os ensaios para a ARC2. Os resultados indicam que os compostos presentes no efluente apresentam custos diferentes para remoção. Essa análise torna-se relevante, pois relaciona a eficiência ao consumo de energia para remoção desse composto (ASHA; KUMAR, 2015a).

Os resultados encontrados nessa análise contrastam com algumas pesquisas que sugerem que maiores níveis de correntes aplicadas tratam o efluente em menor tempo e por isso consomem menos energia elétrica (VALENTE et al., 2015). Nesse ínterim, destaca-se que tais estudos focam apenas na eficiência de remoção e poucos na taxa de remoção. Para Kuokkanen et al. (2013), muitos autores não apresentam valores econômicos relacionados a densidades de correntes em condições ótimas de operação, ao menos por estimativas. Assim, torna-se imprescindível avaliar o consumo de energia em função da taxa de remoção de cada poluente (ASHA; KUMAR, 2015a).

A avaliação do consumo de energia no tratamento de efluentes é um fator decisivo na otimização de sistemas. Valores encontrados na presente pesquisa (Tabela 5) demonstram potencial uso da técnica quando comparado às demais formas de tratamento. Os resultados do consumo geral estão coerentes aos encontrados por Phalakornkule et al. (2010a) para efluentes contendo corantes usando eletrodos de ferro $\left(0,69\right.$ a $\left.1,42 \mathrm{kWh} \mathrm{m}^{-3}\right)$, por Phalakornkule et al. (2010b), para efluentes têxteis usando eletrodos de ferro e alumínio $\left(0,42\right.$ a 1,62 $\left.\mathrm{kWh} \mathrm{m}^{-3}\right)$ e Kobya et al. (2007), que encontraram para têxteis usando eletrodos de alumínio $0,70 \mathrm{kWh} \mathrm{m}^{-3}$, mas pouco superiores aos encontrados para efluentes de curtume usando eletrodos de ferro (KONGJAO et al., 2008) $\left(0,13 \mathrm{kWh} \mathrm{m}^{-3}\right)$. Destaca-se que o uso de eletrodos de ferro apresenta, geralmente, custo inferior quando comparado ao alumínio (KUOKKANEN et al., 2013), devido ao menor valor do material e menor equivalente eletroquímico de dissociação do metal no meio. 
Tabela 5. Consumo geral de energia elétrica (kWh m-3) geral (CEG) e específico para remoção de turbidez (em kWh kNTU ${ }^{-1} \mathrm{~L}^{-1}$ ), Demanda Química de Oxigênio (DQO) e Sólidos Totais (ST) (em kWh kg-1 $\left.\mathrm{L}^{-1}\right)$, e turbidez em cada um dos ensaios

\begin{tabular}{|c|c|c|c|c|c|c|c|c|}
\hline \multirow{3}{*}{ Ensaios } & \multicolumn{4}{|c|}{ ARC1 } & \multicolumn{4}{|c|}{ ARC2 } \\
\hline & \multirow{2}{*}{$\begin{array}{c}\text { CEG } \\
\mathrm{kW} \cdot \mathrm{h} \mathrm{m}^{-3}\end{array}$} & \multirow{2}{*}{$\begin{array}{c}\text { Turbidez } \\
\text { kWh kNTU }^{-1}\end{array}$} & \multirow{2}{*}{\multicolumn{2}{|c|}{$\begin{array}{l}\text { ST } \quad \text { DQO } \\
\mathrm{kWh} \mathrm{kg}^{-1}\end{array}$}} & \multirow{2}{*}{$\begin{array}{c}\text { CEG } \\
\mathrm{kW} \cdot \mathrm{h} \mathrm{m}^{-3}\end{array}$} & \multirow{2}{*}{$\begin{array}{c}\text { Turbidez } \\
\text { kWh kNTU-1 }\end{array}$} & \multirow{2}{*}{\multicolumn{2}{|c|}{$\begin{array}{l}\mathrm{kWh} \mathrm{kg}^{-1} \\
\text { DQO }\end{array}$}} \\
\hline & & & & & & & & \\
\hline 1 & 1,65 & 2,66 & 0,25 & 1,27 & 8,94 & 3,69 & 2,53 & 1,46 \\
\hline 2 & 2,08 & 2,85 & 0,84 & 1,40 & 16,23 & 7,89 & 3,68 & 3,15 \\
\hline 3 & 1,37 & 1,72 & 0,93 & 0,48 & 20,22 & 11,06 & 3,57 & 1,53 \\
\hline 4 & 0,63 & 1,23 & 0,40 & 0,53 & 16,09 & 9,44 & 2,76 & 1,37 \\
\hline 5 & 1,65 & 2,00 & 0,59 & 1,15 & 20,21 & 8,44 & 6,50 & 4,80 \\
\hline 6 & 1,24 & 1,72 & 0,76 & 0,86 & 16,48 & 7,70 & 2,75 & 3,37 \\
\hline 7 & 0,82 & 1,43 & 0,31 & 0,33 & 10,11 & 5,10 & 3,85 & 1,34 \\
\hline 8 & 1,26 & 1,47 & 1,83 & 0,35 & 10,99 & 5,19 & 2,98 & 1,37 \\
\hline 9 & 3,30 & 3,76 & 3,68 & 0,41 & 13,76 & 6,28 & 3,45 & 1,92 \\
\hline 10 & 2,47 & 2,86 & 3,07 & 1,12 & 6,19 & 2,74 & 2,85 & 1,71 \\
\hline 11 & 2,08 & 2,43 & 6,14 & 0,34 & 7,58 & 3,37 & 2,80 & 4,00 \\
\hline 12 & 2,98 & 3,19 & 2,39 & 1,27 & 16,18 & 9,12 & 5,45 & 2,63 \\
\hline
\end{tabular}

A DQO é a variável que possui o consumo de energia mais estudado. Neste estudo encontrou-se valores entre 0,33 a $1,40 \mathrm{kWh} \mathrm{kg}^{-1}$ para a ARC1 e de 1,34 a 4,80 $\mathrm{kWh} \mathrm{kg}^{-1}$ de efluente tratado na ARC2. Os resultados da ARC1 estão próximos aos de Asha e Kumar (2015b) (1,102 kWh kg-1), usando eletrodos de alumínio para tratar ARC e abaixo aos encontrados pelos mesmos autores (ASHA; KUMAR, 2016) (3,61 e $40,0 \mathrm{kWh} \mathrm{kg}^{-1}$ ), quando usaram eletrodos de alumínio e ferro, respectivamente. Para demais efluentes, Apaydin et al. (2009) encontraram $1,8 \mathrm{kWh} \mathrm{kg}^{-1}$ para efluentes de curtume, El-Ashtoukhy e Amin (2010) chegaram a 3,82 $\mathrm{kWh} \mathrm{kg}^{-1}$ para efluentes contendo corante verde ácido, próximos aos encontrados para a ARC2 nesse estudo.

A variável ST esboçou valores entre 0,25 a $6,14 \mathrm{kWh} \mathrm{kg}^{-1}$, na ARC1 e de 2,53 a $6,50 \mathrm{kWh} \mathrm{kg}^{-1}$, na ARC2. Essa diferença se deve à associação entre menores taxas de remoção e maiores DC's aplicadas nos tratamentos. Isso permite aventar que menores eficiências, maiores DC's e TDH's apresentam maiores custos de tratamento (ASHA; KUMAR, 2015a).

Quanto à turbidez, os consumos elétricos ficaram entre 0,63 a 3,30 kWh 
$\mathrm{kNTU}^{-1}$ para a ARC1, e de 2,74 a 11,06 kWh kNTU-1 para ARC2. Terrazes et al. (2010), para remover $92 \%$ da turbidez de efluente da indústria de papel consumiram $0,68 \mathrm{kWh} \mathrm{m}^{-3}$. É importante destacar que são escassos os estudos apresentando resultados de consumo de energia elétrica pela eletrofloculação, principalmente para as variáveis turbidez e sólidos totais.

O custo operacional total do tratamento da ARC1 e ARC2 varia entre 0,25

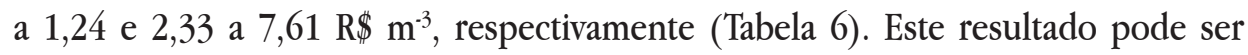
comparado ao estudo de Ferreira (2006), que avaliou o custo de operação de um sistema de EF para tratamento de efluentes de uma indústria de papel. $\mathrm{O}$ valor do custo de operação foi de $\mathrm{R} \$ 2,84 \mathrm{~m}^{-3}$, ficando bem próximo do valor estimado para o presente. Para efluente de laticínio usando eletrodos de ferro, Kushwaha et al. (2010) encontraram um custo do tratamento por EC de aproximadamente R\$5,00 por metro cúbico de efluente tratado para redução de 70\% na DQO.

Tabela 6. Custo de consumo de material, energia elétrica e operacional total para tratamento eletrolítico da Água Residuária do Café sem recirculação (ARC1) e com recirculação (ARC2) em $\mathrm{R} \$ \mathrm{~m}^{-3}$ para cada um dos ensaios conduzidos

\begin{tabular}{|c|c|c|c|c|c|c|c|c|c|}
\hline \multirow{3}{*}{ Ensaios } & \multirow{3}{*}{$\begin{array}{c}\text { Corr } \\
\text { A }\end{array}$} & \multicolumn{4}{|c|}{ ARC1 } & \multicolumn{4}{|c|}{ ARC2 } \\
\hline & & TDH & Material & Energia & Total & TDH & Material & Energia & Total \\
\hline & & $\min$ & & $\mathrm{R} \$ \mathrm{~m}^{-3}$ & & $\min$ & & $\mathrm{R} \$ \mathrm{~m}^{-3}$ & \\
\hline 1 & 2 & 4,12 & 0,13 & 0,49 & 0,62 & 22,34 & 0,70 & 2,66 & 3,36 \\
\hline 2 & 1,5 & 6,87 & 0,16 & 0,62 & 0,78 & 54,10 & 1,27 & 4,84 & 6,11 \\
\hline 3 & 1 & 7,00 & 0,11 & 0,41 & 0,52 & 101,12 & 1,58 & 6,03 & 7,61 \\
\hline 4 & 0,5 & 7,44 & 0,06 & 0,19 & 0,25 & 160,92 & 1,26 & 4,79 & 6,05 \\
\hline 5 & 2 & 4,12 & 0,13 & 0,49 & 0,62 & 50,53 & 1,58 & 6,02 & 7,60 \\
\hline 6 & 1,5 & 4,12 & 0,10 & 0,37 & 0,47 & 54,95 & 1,29 & 4,91 & 6,20 \\
\hline 7 & 1 & 4,12 & 0,06 & 0,25 & 0,31 & 50,53 & 0,79 & 3,01 & 3,80 \\
\hline 8 & 0,5 & 12,62 & 0,10 & 0,38 & 0,48 & 109,89 & 0,86 & 3,27 & 4,13 \\
\hline 9 & 2 & 8,24 & 0,26 & 0,98 & 1,24 & 34,41 & 1,08 & 4,10 & 5,18 \\
\hline 10 & 1,5 & 8,24 & 0,19 & 0,74 & 0,93 & 20,62 & 0,48 & 1,84 & 2,33 \\
\hline 11 & 1 & 10,41 & 0,16 & 0,62 & 0,78 & 37,89 & 0,59 & 2,26 & 2,85 \\
\hline 12 & 0,5 & 29,78 & 0,23 & 0,89 & 1,12 & 161,80 & 1,27 & 4,82 & 6,09 \\
\hline
\end{tabular}


Valente et al. (2015) relatam que não apenas o custo de tratamento deva ser levado em consideração, mas também a eficiência de remoção. Tratando efluente de laticínio, os autores observaram um aumento considerável no custo operacional para um pequeno aumento na remoção de DQO. Para remoção de 50\% de DQO, o custo operacional foi menor que $\mathrm{R} \$ 1,00$ por metro cúbico de efluente tratado. Enquanto para remoção de aproximadamente $70 \%$ na DQO, o custo operacional fica próximo de $R \$ 2,70$ por metro cúbico de efluente tratado.

Alguns autores (BAYRAMOGLU et al., 2004; BAYRAMOGLU et al., 2006; KOBYA et al., 2006) relatam que o maior custo do tratamento por eletroflotação é devido ao consumo de energia elétrica. Essa afirmativa é válida também para o observado no presente estudo. Mesmo com correntes mais baixas $(0,5 \mathrm{~A})$, detectouse custos maiores no consumo de energia elétrica do que de material.

Por fim, releva-se que esse estudo inicial sobre o custo tratamento da Água Residuária do Café (ARC), referente aos elementos de custo operacional total, permite avaliar a possibilidade do emprego dessa tecnologia. Pesquisas com reatores em escalas maiores podem melhorar a estimativa desse custo.

\section{CONSIDERAÇÕES FINAIS}

Por meio do experimento conduzido é possível suplantar que maiores níveis de densidade de correntes conduzem a maiores taxas de remoção de turbidez e sólidos totais e maiores incrementos no $\mathrm{pH}$.

O tempo de detenção e as densidades de correntes são fatores que interferem na remoção da turbidez por apresentar uma coloração indesejada.

A remoção de DQO e seus fatores intervenientes ainda não é completamente compreendido, no entanto entende-se que está relacionado com os sólidos dissolvidos e condições do tratamento eletrolítico.

As variáveis apresentam consumos elétricos diferentes para remoção, estando relacionados à eficiência, tempo e potência aplicada.

A configuração que apresentou o menor consumo geral de energia elétrica foi com a tensão $12 \mathrm{~V}$, Corr 0,5 A, DP $10 \mathrm{~mm}$ e TDH de 7,44 min. 
O custo referente ao consumo de energia é o principal componente do custo operacional, em todos os níveis de correntes aplicados.

\section{REFERÊNCIAS}

ALBARICI, T. R.; FREITAS, D. M.; PESSOA, J. D. C. Protocolos de análise para polpa de açaí: um guia prático de consulta. São Carlos: Embrapa Instrumentação Agropecuária, 2009.

AMERICAN PUBLIC HEALTH ASSOCIATION. Standard Methods For The Examination of Water and Wastewater. 22. ed. Washington: APHA, 2012.

APAYDIN, O.; KURT, U.; GONULLU, M. T. Investigation on the treatment of tannery wastewater by eletrocoagulation. Global Nest Journal, v. 11, n. 4, 2009, p. 546555.

AQUINO NETO, S.; DE ANDRADE, A. R.; Electrochimica. Acta, Maringá, v. 54, n. 2039, 2009.

ASHA, G.; KUMAR, B. M. Coffee pulping wastewater treatment by electrochemical treatment followed anaerobic sequencing batch reactor. International Journal of Scientific \& Engineering Research, v. 6, n. 7, July, 2015a.

ASHA, G.; KUMAR, B. M. Comparison of aluminum and iron electrodes for cod reduction from coffee processing wastewater by electrocoagulation process. Journal of Scientific Research \& Reports, v. 9, n. 7, p. 1-10, 2016.

ASHA, G.; KUMAR, B. M. Evaluation of electrochemical process for treating coffee processing wastewater using aluminum electrodes. Journal of Environmental Science, Toxicology and Food Technology, v. 9, n. 9, II, p. 74-82, sep., 2015 b.

ASSOCIAÇÃO BRASILEIRA DE NORMAS TÉCNICAS - ABNT. Água - Determinação de potássio e sódio: Método da espectrometria de absorção atômica por chama: 13810. Rio de Janeiro, 1997. 3p.

ASSOCIAÇÃO BRASILEIRA DE NORMAS TÉCNICAS - ABNT. Preservação e técni- 
cas de amostragem de efluentes líquidos e corpos receptores: 9898. Rio de Janeiro, 1987. 22p.

BARBOSA, I. M.; TERAN, F. J. C. Eletrocoagulação-floculação aplicada ao tratamento de efluentes gerados na indústria e reciclagem de plásticos. Holos Environment, v. 9 , n. 1, p. 93-112, 2009.

BAYRAMOGLU, M.; KOBYA, M.; CAN, O. T.; SOZBIR, M. Operating cost analysis of electrocoagulation of textile dye wastewater. Separation Purification and Technology, v. 37, p. 117-125, 2004.

BAYRAMOGLU, M.; KOBYA, M.; EYVAZ, M.; SENTURK, E. Technical and economic analysis of electrocoagulation for the treatment of poultry slaughterhouse wastewater. Separation and Purification Technology, v. 51, p. 404-408, 2006.

BENAZZI, T. L.; DALLAGO, R. M.; STEFFENS, J.; BOPSIN, M. H. Effect of ph and conductivity in the electrocoagulation treatment of synthetic dairy effluent. Revista Ciências Exatas e Naturais (Impresso), v. 17, p. 105-118, 2015.

BENAZZI, T. L.; DI LUCCIO, M.; DALLAGO, R. M.; STEFFENS, J.; MORES, R.; NASCIMENTO, M. S.; KREBS, J.; CENI, G. Continuous flow electrocoagulation in the treatment of wastewater from dairy industries. Water Science and Technology, v. 73, p. 1418-1425, 2016.

BORÉM, F. M. Processamento do café. In: BORÉM, F. M. Pós-colheita do café. Lavras: UFLA, 2008. p. 127-158.

BRUNO, M.; OLIVEIRA, R. A. de. Tratamento anaeróbio de águas residuárias do beneficiamento de café por via úmida em reatores UASB. Engenharia Agrícola, Jaboticabal, v. 28, n. 2, p. 364-377, 2008.

BUTLER, E.; HUNG, Y.; YEH, R. Y.; AL AHMAD, M. S. Electrocoagulation in Wastewater Treatment. Water, v. 3, p. 495-525, 2011.

CAMPOS, C. M. M.; PRADO, M. A. C.; PEREIRA, E. L. Caracterização físico-química, bioquímica e energética da água residuária do café processado por via úmida. Biosci. J., Uberlândia, v. 26, n. 4, p. 514-524, July-Aug. 2010. 
CARMONA, M.; KHEMIS, M.; LECLERC, J. P. A simple model to predict the removal of oil suspensions from water using the electrocoagulation technique, J. Chem Eng Sci, v. 6, 2006.

CASILLAS, H. A. M.; COCKE, D. L.; GOMES, J. A. G.; MORKOVSKY, P.; PARGA, J. R.; PETERSON, E. Eletrocoagulation mechanism for COD removal. Sep. Purif. Technol., v. 56, p. 204-211, 2007.

CEMPRE - Compromisso Empresarial Para a Reciclagem. Mercado - Preço de material reciclável. (2017) Disponível em: http://cempre.org.br/servico/mercado.

CERQUEIRA, A. A.; SOUZA, P. S. A.; MARQUES, M. R. C. Effects of direct and alternating current on the treatment of oily water in an electroflocculation process. Brazilian Journal of Chemical Engineering, v. 31, n. 03, p. 693-701, Jul-Sep., 2014.

CERQUEIRA, A.; RUSSO, C.; MARQUES, M. R. C. Electroflocculation for textile wastewater treatment. Brazilian Journal of Chemical Engineering, v. 26, n. 04, p. 659-668, oct.-dec., 2009.

CETESB. Companhia Ambiental do Estado de São Paulo. Significado ambiental e sanitário das variáveis de qualidade das águas e dos sedimentos e metodologias analíticas de amostragem. Apêndice A, Série de relatórios. Secretaria de Meio Ambiente, Governo do Estado de São Paulo, 2008.

CHEN, X.; CHEN, G.; YUE, P. L. Separation of pollutants from restaurant wastewater by electrocoagulation, Sep. Purif. Technol., v. 19, 2000.

CHEN, G. Electrochemical technologies in wastewater treatment. Separation and Purification Technology, v. 38, p. 11-41, 2004.

EL-ASHTOUKHY, E. Z.; AMIN, N. K. Removal of Acid Green Dye 50 from Wastewater by Anodic Oxidation and Electrocoagulation - A Comparative Study. Journal of Hazardous Materials, v. 179, n. 1-3, 2010, p. 113-119.

ESCELSA - Centrais Elétricas do Espírito Santo S. A. Resolução Homologatória $\mathrm{n}^{\mathrm{O}} 2203$ de 14.02.2017, Estabelece as faixas de acionamento e os adicionais das Bandeiras tarifárias, a partir de fevereiro de 2017. Homologa as Tarifas de Energia 
- TE e as Tarifas de Uso dos Sistemas de Distribuição - TUSD referentes à Espírito Santo Centrais Elétricas S. A. Espírito Santo, Brasil, 3p.

FIA, R.; MATOS, A. T.; MATOS, M. P.; ABREU, E. C.; FIA, F. R. L. Tratamento das águas do processamento dos frutos do cafeeiro em filtro anaeróbio seguido por sistema alagado construído: I - Remoção de matéria orgânica. Eng. Agríc., Jaboticabal, v. 30, n. 6, p. 1191-1202, 2010d.

FIA, R.; MATOS, A. T.; QUEIROZ, M. E. L. R. de.; CECON, P. R.; FIA, F. R. L. Desempenho de sistemas alagados no tratamento de águas residuárias do processamento dos frutos do cafeeiro. Revista Brasileira de Engenharia Agrícola e Ambiental, Campina Grande, v. 14, n. 12, p. 1323-1329, 2010a.

FIA, R.; MATOS, A. T.; LAMBERT, T. F.; FIA, F. R. L.; MATOS, M. P. Tratamento das águas do processamento dos frutos do cafeeiro em filtro anaeróbio seguido por sistema alagado construído: II - Remoção de nutrientes e compostos fenólicos. Eng. Agríc, Jaboticabal, v. 30, n. 6, p. 1203-1213, 2010e.

FERREIRA, L. H. Remoção de sólidos em suspensão de efluente da indústria de papel por Eletroflotação. (Dissertação de mestrado). Unicamp, Campinas, 2006.

GOLDER, A. K.; HRIDAYA, N.; SAMANTA, A. N. Electrocoagulation of methylene blue and eosin yellowish using mild steel electrodes. J. J Hazard Mater, v. 127, 2005.

HOLT, P. K.; BARTON, G. W.; MITCHELL, C. A. The future for electrocoagulation as a localised water treatment technology. Chemosphere, v. 59, p. 355-367, 2005.

INAN, H.; DIMOGLO, A.; SIMSEK, H.; KARPUZCU, M. Olive oil mill wastewater treatment by means of electro-coagulation. Elsevier B.V. Separation and Purification Technology, v. 36, 2004.

KABDA LI, I.; ARSLAN-ALATON, I.; ÖLMEZ-HANCI, T.; TÜNAY, O. Electrocoagulation applications for industrial wastewaters: a critical review. Environmental Technology Reviews, v. 1, n. 1, p. 2-45, 2012. 
KANNAN, N.; KARTHIKEYAN, G.; TAMILSELVAN, N. Comparison of treatment potentil of electrocoagulation of distillery effluent with and without activated Areca catechu nut carbon. Elsevier B. V. Journal of Hazardous Materials, v. 137, 2006.

KOBYA, M.; DEMIRBAS, E.; CAN, O. T. Treatment of levafix orange textile dye solution by electrocoagulation. J. Hazard Mater, v. 132, $2006 \mathrm{~b}$.

KOBYA, M.; HIZ, H.; SENTURK, E.; AYDINER, C.; DEMIRBAS, E. Treatment of potato chips manufacturing wastewater by electrocoagulation. Desalination, v. 190, $2006 a$.

KOBYA, M.; BAYRAMOGLU, M.; EYVAZ, M. Technoeconomical evaluation of electrocoagulation for the textile wastewater using different electrode connections. J. Hazard. Mater. v. 148, 2007, p. 311-318.

KONDO, M. M.; MORAES, R. G. M.; ANDRADE, S. J.; SILVA, M. R. A. Processos fenton e foto-fenton no tratamento de águas dos frutos do cafeeiro. Coffee Science, Lavras, v. 9, n. 4, p. 506-515, 2014.

KONGJAO, S.; DAMRONGLERD, S.; HUNSOM, M. Simultaneous Removal of Organic and Inorganic Pollutants in Tannery Wastewater Using Electrocoagulation Technique. Korean Journal of Chemical Engineering, v. 25, n. 4, 2008, p. 703-709.

KUOKKANEN, V.; KUOKKANEN, T.; RÄMÖ, J.; LASSI, U. Recent applications of electrocoagulation in treatment of water and wastewater - A Review. Green and Sustainable Chemistry, v. 3, p. 89-121, 2013.

KUSHWAHA, J. P.; SRIVASTAVA, V. C.; MALL, I. D. Organics removal from dairy wastewater by eletrochemical treatment and residual disposal. Separation and Purification Technology, v. 76, p. 198-205, 2010.

MATOS, A. T.; CABANELLAS, C. F. G.; CECON, P. R.; BRASIL, M. S.; MUDADO, C. S. Efeito da concentração de coagulantes e do pH da solução na turbidez da água, em recirculação, utilizada no processamento dos frutos do cafeeiro. Eng. Agríc., Jaboticabal, v. 27, n. 2, p. 544-551, maio/ago. 2007.

MATOS, A. T.; EUSTÁQUIO JÚNIOR, V.; MATOS, M. P. de. Eficiência de aeração e 
consumo de oxigênio no tratamento de água residuária do processamento dos frutos do cafeeiro em sistema de aeração em cascata. Eng. Agríc., Jaboticabal, v. 35, n. 5, p. 941-950, set./out. 2015.

MOLLAH, M. Y. A.; MORKOVSKY, P.; GOMES, J. A. G.; KESMEZ, M.; PARGA, J.; COCKE, D. L. Fundamentals, present and future perspectives of electrocoagulation. J. Hazard. Mater., 2004.

OLIVEIRA, R. A.; BRUNO, N. M. N. Start-up of horizontal anaerobic reactors with sludge blanket and fixed bed for wastewater treatment from coffee processing by wet method. Eng. Agríc., Jaboticabal, v. 33, n. 2, p. 353-366, 2013.

PERALTA, Y. M.; SANABRIA, N. R.; CARRIAZO, J. G.; MORENO, S.; MOLINA, R. Catalytic wet hydrogen peroxide oxidation of phenolic compounds in coffee wastewater using Al-Fe-pillared clay extrudates. Desalination and Water Treatment. may 2014.

PHALAKORNKULE, C.; POLGUMHANG, S.; TONGDAUNG, W.; KARAKAT, B.; NUYUT, T. Electrocoagulation of Blue Reactive, Red Disperse and Mixed Dyes, and Application in Treating Textile Effluent. Journal of Environmental Management, v. 91, n. 4, 2010b, p. 918-926.

PHALAKORNKULE, C.; SUKKASEM, P.; MUTCHIMSATTHA C. Hydrogen Recovery from the Electrocoagulation Treatment of Dye-Containing Wastewater. International Journal of Hydrogen Energy, v. 35, n. 20, 2010a, p. 10934-10943.

SELVAMURUGAN, M.; DORAISAMY, P.; MAHESWARI, M.; NANDAKUMAR, N. B. High rate anaerobic treatment of coffee processing wastewater using upflow anaerobic hybrid reactor. Iranian Journal Environmental Health Science and Engineering, v. 7, p. 129-136, 2010.

SONG, Z.; WILLIAMS, C. J.; EDYVEAN, R. G. J. Treatment of tannery wastewater by chemical coagulation. J. Desalination, v. 13, 2004.

TCHAMANGO, S.; NANSEU-NJIKI, C. P.; NGAMENI, E.; HADJIEV, D.; DARCHEN, A. Treatment of dairy effluents by electrocoagulation using aluminium electrodes. Elsevier B. V. Science of the Total Environment, v. 408, 2010. 
TERRAZAS, E.; VÁZQUEZ, A.; BRIONES, R.; LÁZARO, I.; RODRÍGUEZ, I. EC. Treatment for reuse of tissue paper wastewater: Aspects that affect energy consumption. J. Hazard. Mater. 2010, 181, 809-816.

VALENTE, G. F. S. Avaliação da eletrocoagulação no tratamento de efluente líquido da indústria de laticínios. $117 \mathrm{f}$. Tese (Doutorado em Ciência e Tecnologia de Alimentos). Universidade Federal de Viçosa, Viçosa. 2012.

VALENTE, G. F. S.; MENDONÇA, R. C. S.; PEREIRA, J. A. M.; FELIX, L. B. The efficiency of electrocoagulation in treating wastewater from a dairy industry, Part I: Iron electrodes. Journal of Environmental Science and Health. v. 47, p. $355-$ 361, 2012.

VALENTE, G. F. S.; MENDONÇA, R. C. S.; PEREIRA, J. A. M. The efficiency of electrocoagulation using aluminum electrodes in treating wastewater from a dairy industry. Ciência Rural, v. 45, p. 1713-1719, 2015.

Recebido em: 06/03/2018

Aceito em: 24/05/2018 